\title{
Culture-Positivity and Sensitivity in Post-Surgical Patients in a Tertiary Care Hospital
}

${ }^{1}$ Ehsaan Ahmed, ${ }^{2}$ Muhammad Imran Anwar, ${ }^{1}$ Ruqaya Idrees, ${ }^{1}$ Sheza Arif Toor,

${ }^{1}$ Asad Mahmood, ${ }^{1}$ Ayesha Humayun

${ }^{1}$ Department of Public Health \& Community Medicine, SKZMC, Shaikh Zayed Medical Complex, Lahore

${ }^{2}$ Department of Surgery, Shaikh Zayed Medical Complex, Lahore

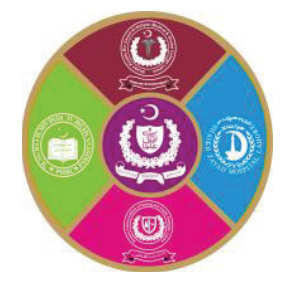

\begin{abstract}
Introduction: Hospital-Acquired Infection (HAI) is a serious health hazard across the world, being a major contributor to the rate of morbidity and mortality. Inadequate evidence is available on the magnitude of hospital-acquired infections in tertiary care hospitals of Pakistan, thus resulting in a poor focus on this aspect of quality of care. Aims \& Objectives: The study aims to measure the frequency of post-op culture-positivity, sensitivity, and its associated attributes in a tertiary care hospital in Lahore, Pakistan. Place and duration of study: Tertiary care teaching hospital in Lahore, Punjab from April to July, 2016. Material \& Methods: A cross-sectional study was conducted in a public sector, tertiary care, teaching hospital in Lahore using secondary data. All discharged patients' files from surgical and allied specialties, operated from $1^{\text {st }}$ Oct, 2015 to $31^{\text {st }}$ Dec 2015, having ages between 18 to 60 years and of both gender, were included consecutively in the study. Data was collected on a structured, self-constructed form and analyzed through SPSS version 21. Results: Out of the total 420 patient files, $47(11.2 \%)$ had culture positive reports, out of which $74.5 \%$ had UTIs, $17 \%$ tissue/wound infection, $4.3 \%$ fluid infections and $4.3 \%$ had other site infections. $46 \%$ of the females and $32 \%$ of the males were found to be culture positive out of the suspects of HAI. The highest infection rate was found among patients of urology (71\%), while there was no positive culture found for neurosurgery patients. The most frequently found organisms in cultures were Coliform species (38\%), Pseudomonas Aeruginosa (13\%), followed by Escherichia coli, Staphylococcus Aureus and Candida Albican. Mixed growth was found in $26 \%$ of the cultures. The antibiotic sensitivity of the cultures was to Amikacin (38.3\%), Imipenem (29.8\%), Tazocin (21.3\%) and Gentamycin (14.9\%). The antibiotic resistance among the cultures was to; Ciproxin (44.7\%), Amoxicillin (34.0\%), Cefotaxime (31.9\%) and Ampicillin (23.4\%). Conclusion: The infection rate among suspects of HAI, was found to be high in this study which calls for an urgent need of robust infection control policy and strategies.
\end{abstract}

Key words: Hospital acquired infections, nosocomial infections, culture-positivity, culture sensitivity

\section{INTRODUCTION}

$\mathrm{H}$ ospital acquired infection (HAI) is a serious health hazard across the world. World Health Organization (WHO) described it as one of the major threats posing huge economic impact. Nosocomial infections, hospital acquired infections and health-care associated infections are terms used interchangeably. Despite much advances in the control and prevention of hospital acquired infections, still continue to remain major contributor to the rate of morbidity, mortality and cost of care. ${ }^{1,2}$ Nosocomial infections can be defined as those occurring within 48 hours of hospital admission, 3 days of discharge or 30 days of a surgery.
Postoperative nosocomial infection is a surgical site or blood stream infection occurring within 48 hours of surgery until the time of discharge from hospital with clinical signs and symptoms and laboratory confirmation. ${ }^{3}$ The signs of infection include pain, tenderness, warmth, erythema, swelling and discharge. ${ }^{4}$ Prevalence of HAI is 9.4 percent in the developed countries. In a meta analysis and systematic review on health-care-associated infections reported ICU pooled densities at least three times higher than from USA. Surgical-site infection was leading with proportions higher than reported in developed countries. Gram-negative bacilli are commonly identified with methicillin resistant Staphylococcus aureus. ${ }^{5}$ Surgical services constitute a large portion of hospital care with wide 
range of major and minor operative procedures being conducted at a tertiary care setup. Postoperative period is of great concern while looking at surgical services as a whole. The primary objective of postoperative care is to minimize patient's discomfort, assist restitution of health and prevent infections. ${ }^{6,7}$ Infection of surgical wounds resulting in pyrexia, discomfort, serious complications and subsequent prolonged use of antimicrobials are quite common in our part of the world. The source of these infections can be exogenous or endogenous bacteria. This has a grave impact on patient's overall health and finances and a burden on hospitals.

Conventionally prophylactic antibiotics are prescribed to the patient both before and after the surgery. Duration and type of prophylactic antibiotics received by the patient matters a lot in the development of postoperative infections along with patient factors as well. ${ }^{8}$ The increasing poly antimicrobial resistance amongst hospital pathogens also poses a great threat. ${ }^{9}$ Usually wound, blood, sputum, fluid, stool and urine samples are sent for culture testing to identify microbial presence, growth as well as sensitivity and resistance to specific antibiotics. ${ }^{10,11}$ Postoperative infections result in prolonged duration of hospital stay and pose economic, physical and psychological burden on the patient and the entire family. This raises serious questions about hospital's performance, standards, hygiene, maintenance and economics. ${ }^{12}$ Postoperative infections are good indicators for hospital associated infections (nosocomial infections) and quality of hospital care. Multiple factors influence infection control behaviors and practices in surgery therefore interventions are needed to ensure shared ownership of all clinical outcomes. ${ }^{13}$

Quality assurance and control activities are the backbone of hospital services so evidence-informed guidelines are greatly needed to provide new and updated evidence-based recommendations for the prevention of SSI and should be incorporated into comprehensive surgical quality improvement programs to improve patient safety. ${ }^{14}$ Healthcare expenditure in Organization for Economic Cooperation and Development (OECD) countries is increasing due to increased pharmaceutical expenditure, hospital care and the high prevalence of chronic diseases and their financial consequence. ${ }^{15,16,17}$ This has created a need to implement systems to improve the efficiency and quality of health care ${ }^{18}$ which is the reason behind this study.
An Iranian study showed $60.6 \%$ antimicrobial resistance in nosocomial infection cases. ${ }^{19}$ In Pakistan the frequency of nosocomial infection in ICU of a tertiary care hospital was reported $29.13 \%$ in $2008^{20}$ and Surgical site infection in post surgical patients was $7.3 \%$ in another hospital in $2011 .{ }^{21} \mathrm{~A}$ multi-centered study from Pakistan showed a Acinetobacter baumannii, the most prominent antibiotic-resistance phenotype. ${ }^{22}$

Alarmingly, we are moving towards pre-antibiotic era very fast and hospitals are a source of antimicrobial resistance. ${ }^{23}$ This study was urgently much needed to highlight the issue so the findings can be taken up to develop contextualized, evidence-informed hospital policies. Recent quality literature from Pakistan on the magnitude of nosocomial infections in surgical and allied services of tertiary care hospitals is limited. Current study will guide administrative policies guidelines and plans for infection control in surgical settings and services. Current study is aimed at measuring the frequency of postoperative culture positivity and sensitivity at one of the largest tertiary care hospitals in Lahore.

\section{MATERIAL AND METHODS}

A descriptive cross-sectional study was conducted at a tertiary care teaching hospital in Lahore, Punjab from April to July, 2016 on secondary data of patients who were operated upon during 1st October to $31^{\text {st }}$ December, 2015. This hospital houses more than 1000 beds.

\section{Eligibility Criteria:}

All patient files, all those operated upon from 1st October 2015 to $31^{\text {st }}$ December 2015 in General Surgery or the allied specialties except Gynecology and Obstetrics were our study population. Researchers had constraints of time so they only conducted this study on 3 months record therefore this project was limited by time convenience.

Patients who had been re-operated within 30 days of first operation, had developed a complication or were declared immune-compromised were not included in the study. Tampered files and files with inadequate data were also not included.

The data was collected from the medical record room of the hospital by the research team maintaining anonymity and confidentiality of data. The patients' records were used to fill the selfconstructed questionnaire designed specifically to acquire information about culture indicated, culture positivity and sensitivity to the available antibiotics. For this purpose, the microbiology reports of the specimens sent (including but not restricted to: 
blood, sputum, urine, stool, wound/tissue and fluids) to the microbiology laboratory of the hospital were used. Current study assessed the frequency of culture positivity and sensitivity according to specialty, type of organisms identified, antibiotics sensitivity and resistance along with gender-wise distribution of these variables.

\section{Statistical analysis:}

The data was entered and analyzed in the IBM SPSS version 21 software.

\section{RESULTS}

Total patients discharged during the study period were 420 , out of which $41.9 \%$ were females and $58.1 \%$ were males. Out of $420,58.4 \%$ patients were operated in general surgery and $41.6 \%$ patients in allied surgical specialties (mentioned in Inclusion criteria). Average duration of stay of 420 patients was 9.92 days with $\mathrm{SD}+8.37$ and range of 1 to 55 days. Overall $268 / 420 \quad(63.8 \%)$ patients had prolonged stay ( $>5$ days) and 152/420 (36.2\%) had 5 days or less post-op stay at the hospital. The usual practice is to give post operative antibiotic cover and those patients with symptoms and signs of HAI are investigated by sending samples for culture sensitivity. 124/420 (29.5\%) were suspected to have acquired infection during hospital stay (as per their record files) so, samples were sent of $40.3 \%$ females and $59.7 \%$ males for culture sensitivity. Out of total 420 patients operated, $47(11.19 \%)$ were culture positive during hospital stay, for acquired infection. Out of 124 cultures indicated, 47 (37.9\%) were found to be positive while $77(62.1 \%)$ were culture negative. $51.1 \%$ of our culture positives were male patients and $48.9 \%$ females. $64.9 \%$ of the total culture negatives were males and $35.1 \%$ females. There was no significant difference $(\mathrm{P}=>0.05)$.

Out of culture positives (47/124), Urinary Tract Infections (UTIs) was leading the list with 35 out of $47(74.5 \%)$ and tissue/ wound infection was second with 8 out of $47(17.0 \%)$, blood/ fluid infection was $2 / 47(4.3 \%)$ and other site infections were 2 (4.3\%). Distribution of culture indicated/sent and culture positivity according to type of surgical specialty showed that; Urology cultures sent were 93/124 $(75 \%)$ out of which 33 were positive. In general surgery, 18/124 cultures were sent showing 8 positive. In Orthopedics, 9/124 (7.26\%) cultures were sent with 4 positive. In CTS, 4/124 (3.22\%) cultures were sent out of which $50 \%$ were positive. In Neuro-Surgery, $1 / 124(0.81 \%)$ culture was sent during the period of study which came back negative. Department of Urology was the highest and Neuro-surgery being the lowest among them.

Antibiotic sensitivity results showed that 38.9\% (18/47) of positive cultures were sensitive to Amikacin, 29.8\% (14/47) of positive cultures were sensitive to Imipenem, $21.3 \%$ (10/47) of positive cultures were sensitive to Tazocin and 14.9\% (7/47) of positive cultures were sensitive to Gentamycin.

Antibiotic resistance results showed that $44.7 \%$ (21/47) of positive cultures were resistant to Ciproxin, $34.0 \%(16 / 47)$ of positive cultures were sensitive to Amoxicillin, 31.9\% (15/47) of positive cultures were resistant to Cefotaxime and $23.4 \%$ (11/47) of positive cultures were resistant to Ampicillin.

The most frequently found organisms in cultures were Coliform species (38\%), Pseudomonas Aeruginosa (13\%), Escherichia coli (10\%), Staphylococcus Aureus (8\%) and Candida Albicans $(5 \%)$. Mixed growth was found in $26 \%$ of the cultures (Above data in Table-1).

\begin{tabular}{|l|l|l|}
\hline Variables & Frequency & Percentage \\
\hline Gender & $(\mathrm{n}=47)$ & \\
\hline Males & 24 & $51.1 \%$ \\
\hline Females & 23 & $48.9 \%$ \\
\hline Type of Infection & $(\mathrm{n}=47)$ & \\
\hline UTI & 35 & $74.5 \%$ \\
\hline Tissue/wound & 8 & $17 \%$ \\
\hline Blood/fluid & 2 & $4.3 \%$ \\
\hline Other sites & 2 & $4.3 \%$ \\
\hline Specialties & $(\mathrm{n}=47)$ & \\
\hline Urology & 33 & $71 \%$ \\
\hline General Surgery & 8 & $17 \%$ \\
\hline Orthopedics & 4 & $8.5 \%$ \\
\hline Cardiothoracic & 2 & $4.2 \%$ \\
\hline Neurosurgery & 0 & $0 \%$ \\
\hline Antibiotic sensitivity & $(\mathrm{n}=47)$ & \\
\hline Amikacin & 18 & $38.9 \%$ \\
\hline Imipenem & 14 & $29.8 \%$ \\
\hline Tazocin & 10 & $21.3 \%$ \\
\hline Gentamycin & 7 & $14.9 \%$ \\
\hline Antibiotic resistance & $(\mathrm{n}=47)$ & \\
\hline Ciproxin & 21 & $44.7 \%$ \\
\hline Amoxicillin & 16 & $34 \%$ \\
\hline Cefotaxime & 15 & $31.9 \%$ \\
\hline Ampicillin & 11 & $23.4 \%$ \\
\hline Microorganism growth & $(\mathrm{n}=47)$ & \\
\hline Coliform & 18 & $38 \%$ \\
\hline Pseudomonas A. & 6 & $13 \%$ \\
\hline Escherichia coli & 5 & $10 \%$ \\
\hline Staphlococcus A. & 4 & $5 \%$ \\
\hline Candida A. & 2 & $26 \%$ \\
\hline Mixed growth & 12 & \\
\hline Table-1: Cutte sen & \\
\hline
\end{tabular}

Table-1: Culture sensitivity and resistance pattern among post-surgical culture positive samples in a tertiary care hospital 


\section{DISCUSSION}

Our results provide evidence that nosocomial infections are a serious problem in tertiary care hospitals in Pakistan, since the rates found are high $(11.19 \%)$. In a systematic review conducted on healthcare associated infections in Africa showed a hospital-wide prevalence of $2.5 \%$ to $14.8 \%$ in surgical wards, surgical site infection(SSI) was the main focus. ${ }^{24}$ Rates of current study are within this range and near to the maximum limit found in literature from Africa, while it is more than another systematic literature review and meta-analysis of 41 studies, on the burden of healthcare-associated infections (HAIs) in Southeast Asia, showing a pooled prevalence of overall HAIs as $9.0 \% .{ }^{25}$ In literature from Pakistan, the nosocomial infection rate of ICU was reported to be $29.13 \%$ in $2008^{20}$ while Surgical Site Infection (SSI) rate was $7.3 \%$ in 2011. ${ }^{21}$ Nosocomial infection surveillance and its epidemiology are found to be well studied in literature over last decade in different operative patients. $^{26,27,28}$

In current study, average duration of stay was 9.92 days with $\mathrm{SD} \pm 8.37$ and range of 1 to 55 days. $63.8 \%$ patients had prolonged stay ( $>5$ days). This is different from the findings of a study, where SSI rate reported was $9.85 \%$, the median time to SSI was the 7th postoperative day and the average length of stay was $4.2 \pm 3.4$ days. ${ }^{29}$

In present study findings, amongst the post-op positive culture reports, urine culture was $74.5 \%$ followed by tissue/ wound infection/SSI $17.0 \%$. In a study of 2019, from a hospital in Pakistan, done on post-operative patients, reported SSI rate as $33.68 \%$ which is very high as compared to our study findings. ${ }^{30}$

In current study resistant strains were mostly sensitive to Amikacin followed by imipenem, Tazocin and and Gentamycin while antibiotic resistance was found mainly to Ciproxin, followed by Amoxicillin and Cefotaxime. In our study, Coliform species were most frequently found (31.9\%) followed by Pseudomonas Aeruginosa, Escherichia coli, Staphylococcus Aureus and Candida Albicans. Mixed growth was found in 26\% of the cultures. In a study in India, Escherichia coli was most commonly isolated microbe followed by Klebsiella pneumoniae, Pseudomonas aeruginosa, Enterococcus faecalis, Staphlococcus aureus and Coagulase negative staphylococci. ${ }^{31}$ Klebsiella spp. was the most frequently $(27 \%)$ isolated bacterium in another study in $2016 .{ }^{11}$ In a systematic review and meta-analysis published in 2019 on 66 studies, Escherichia coli, Klebsiella pneumoniae and Staphylococcus spp were reported in majority of studies. ${ }^{32}$

In the age of antibiotics, nosocomial infections are still uncontrollable. Implementation of Infection control practices through continuous training and monitoring of health care providers could help in reducing nosocomial infection rates. Irrational use of antibiotics is an important cause of drug-resistant organisms. Nosocomial surveillance in hospital needs to be stringent followed by adequate measures to address deficiencies in tertiary care health setups in countries like Pakistan.

Limitations in current research include the data extraction from manual record keeping system of patient files. Surgical and medical specialties and different hospitals could be compared to get a larger evidence for development and revision of uniform infections control policies in hospitals of Pakistan. Qualitative case studies can give in-depth insight into the problem and solutions of HAI.

\section{CONCLUSION}

Our study found high frequency of culture positivity in all suspects of nosocomial infections in different surgical specialties of a tertiary care hospital. Antimicrobial resistance was high and this is found to be consistent with local and international literature.

\section{REFERENCES}

1. Ahmed MI. Prevalence of nosocomial wound infection among postoperative patients and antibiotics patterns at teaching hospital in Sudan. North American journal of medical sciences. 2012; 4(1):29.

2. Jarvis WR. Selected aspects of the socioeconomic impact of nosocomial infections: morbidity, mortality, cost, and prevention. Infection Control \& Hospital Epidemiology. 1996; 17(08):552-7.

3. Mulu W, Kibru G, Beyene G, Damtie M. Postoperative Nosocomial Infections and Antimicrobial Resistance Pattern of Bacteria Isolates among Patients Admitted at Felege Hiwot Referral Hospital, Bahirdar, Ethiopia. Ethiopian Journal of Health Sciences. 2012; 22(1):7-18.

4. Cutting KF, White R. Defined and refined: criteria for identifying wound infection revisited. British journal of community nursing. 2004; 9(3):S6-15.

5. Allegranzi B, Bagheri Nejad S, Combescure C, Graafmans W, Attar H, Donaldson L, et al. 
Burden of endemic health-care-associated infection in developing countries: systematic review and meta-analysis. Lancet (London, England). 2011; 377(9761):228-41.

6. Stulberg JJ, Delaney CP, Neuhauser DV, Aron $\mathrm{DC}, \mathrm{Fu} \mathrm{P}$, Koroukian SM. Adherence to surgical care improvement project measures and the association with postoperative infections. Jama. 2010; 303(24):2479-85.

7. Awad SS. Adherence to surgical care improvement project measures and postoperative surgical site infections. Surgical infections. 2012; 13(4):234-7.

8. Flores-Mireles A, Hreha TN, Hunstad DA. Pathophysiology, Treatment, and Prevention of Catheter-Associated Urinary Tract Infection. Top Spinal Cord Inj Rehabil. 2019;25(3):228-40

9. Huang $\mathrm{CH}$, Chiu $\mathrm{CH}$, Chen IW, Hung SY, Lin $\mathrm{CW}$, Hsu BR, et al. Antimicrobial resistance and outcomes of community-onset bacterial bloodstream infections in patients with type 2 diabetes. J Glob Antimicrob Resist. 2018; 15:271-6.

10. Satti MZ, Hamza M, Sajid Z, Asif O, Ahmed H, Zaidi SMJ, et al. Compliance Rate of Surgical Antimicrobial Prophylaxis and its Association with Knowledge of Guidelines Among Surgical Residents in a Tertiary Care Public Hospital of a Developing Country. Cureus. 2019; 11(5):e4776

11. Nouetchognou JS, Ateudjieu J, Jemea B, Mesumbe EN, Mbanya D. Surveillance of nosocomial infections in the Yaounde University Teaching Hospital, Cameroon. BMC Res Notes. 2016; 9(1):505.

12. Zimlichman E, Henderson D, Tamir O, Franz C, Song P, Yamin CK, et al. Health care-associated infections: a meta-analysis of costs and financial impact on the US health care system. JAMA Intern Med. 2013; 173(22):2039-46.

13. Troughton R, Mariano V, Campbell A, Hettiaratchy S, Holmes A, Birgand G. Understanding determinants of infection control practices in surgery: the role of shared ownership and team hierarchy. Antimicrob Resist Infect Control. 2019; 8:116.

14. Berríos-Torres SI, Umscheid CA, Bratzler DW, Leas B, Stone EC, Kelz RR, et al. Centers for Disease Control and Prevention guideline for the prevention of surgical site infection. JAMA surgery. 2017; 152(8):784-91.

15. Armstrong BK, Gillespie JA, Leeder SR, Rubin GL, Russell LM. Challenges in health and health care for Australia. The Medical journal of Australia. 2007; 187(9):485-9.
16. Lorenzoni L, Belloni A, Sassi F. Health-care expenditure and health policy in the USA versus other high-spending OECD countries. The Lancet. 2014; 384(9937):83-92.

17. Squires DA. Explaining high health care spending in the United States: an international comparison of supply, utilization, prices, and quality. Issue brief (Commonwealth Fund). 2012; 10:1-14.

18. Hoque DM, Kumari V, Ruseckaite R, Romero L, Evans SM. Impact of clinical registries on quality of patient care and health outcomes: protocol for a systematic review. BMJ open. 2016; 6(4): 010654.

19. Rajabi M, Abdar ME, Rafiei H, Aflatoonia MR, Abdar ZE. Nosocomial Infections and Epidemiology of Antibiotic Resistance in Teaching Hospitals in South East of Iran. Glob J Health Sci. 2015; 8(2):190-7.

20. Shaikh JM, Devrajani BR, Shah S, Akhund T, Bibi I. Frequency, pattern and etiology of nosocomial infection in intensive care unit: an experience at a tertiary care hospital. J Ayub Med Coll Abbottabad. 2008; 20(4):37-40.

21. Bibi S, Channa GA, Siddiqui TR, Ahmed W. Frequency and risk factors of surgical site infections in general surgery ward of a tertiary care hospital of Karachi, Pakistan. Int J Infect Control. 2011; 7(3):5.

22. Hasan B, Perveen K, Olsen B, Zahra R. Emergence of carbapenem-resistant Acinetobacter baumannii in hospitals in Pakistan. J Med Microbiol. 2014; 63(Pt 1):50-5.

23. Michael CA, Dominey-Howes D, Labbate M. The antimicrobial resistance crisis: causes, consequences, and management. Frontiers in public health. 2014; 2:145.

24. Nejad SB, Allegranzi B, Syed SB, Ellis B, Pittet D. Health-care-associated infection in Africa: a systematic review. Bulletin of the World Health Organization. 2011; 89:757-65.

25. Ling ML, Apisarnthanarak A, Madriaga G. The Burden of Healthcare-Associated Infections in Southeast Asia: A Systematic Literature Review and Meta-analysis. Clin Infect Dis. 2015; 60(11):1690-9.

26. Iordanou S, Middleton N, Papathanassoglou E, Raftopoulos V. Surveillance of device associated infections and mortality in a major intensive care unit in the Republic of Cyprus. BMC Infect Dis. 2017; 17(1):607.

27. Gomez-Lopez R, Barge-Caballero E, Fernandez-Ugidos P, Paniagua-Martin MJ, Barge-Caballero G, Couto-Mallon D, et al. InHospital Post-Operative Infection after Heart 
Transplantation: Epidemiology, Clinical Management, and Outcome. Surg Infect (Larchmt). 2019.

28. Curcio D, Cane A, Fernandez F, Correa J. Surgical site infection in elective clean and clean-contaminated surgeries in developing countries. Int J Infect Dis. 2019; 80:34-45.

29. Zejnullahu VA, Isjanovska R, Sejfija Z, Zejnullahu VA. Surgical site infections after cesarean sections at the University Clinical Center of Kosovo: rates, microbiological profile and risk factors. BMC Infect Dis.2019;19(1):752

30. Sattar F, Sattar Z, Zaman M, Akbar S. Frequency of Post-operative Surgical Site Infections in a Tertiary Care Hospital in Abbottabad, Pakistan. Cureus. 2019; 11(3).

31. Ginawi I, Saleem M, Sigh M, Vaish AK, Ahmad I, Srivastava VK, et al. Hospital acquired infections among patients admitted in the medical and surgical wards of a nonteaching secondary care hospital in northern India. J Clin Diagn Res. 2014; 8(2):81-3.

32. Mouiche MMM, Moffo F, Akoachere JTK, Okah-Nnane NH, Mapiefou NP, Ndze VN, et al. Antimicrobial resistance from a one health perspective in Cameroon: a systematic review and meta-analysis. BMC Public Health. 2019; 19(1):1135.

\section{The Authors:}

Dr. Ehsaan Ahmed

Research Assistant (Honorary),

Department of Public Health \& Community Medicine, Shaikh Khalifa Bin Zayed Al-Nahyan Medical College, Shaikh Zayed Medical Complex, Lahore.
Prof. Muhammad Imran Anwar

Department of Surgery,

Shaikh Zayed Medical Complex, Lahore.

Dr. Ruqaya Idrees

Research Assistant (Honorary),

Department of Public Health \& Community Medicine,

Shaikh Khalifa Bin Zayed Al-Nahyan Medical

College, Shaikh Zayed Medical Complex, Lahore.

Dr. Sheza Arif Toor

Research Assistant (Honorary),

Department of Public Health \& Community Medicine,

Shaikh Khalifa Bin Zayed Al-Nahyan Medical

College, Shaikh Zayed Medical Complex, Lahore.

Dr. Asad Mahmood

Research Assistant (Honorary),

Department of Public Health \& Community Medicine,

Shaikh Khalifa Bin Zayed Al-Nahyan Medical

College, Shaikh Zayed Medical Complex, Lahore.

Prof. Ayesha Humayun

Department of Public Health \& Community Medicine, Shaikh Khalifa Bin Zayed Al-Nahyan Medical College, Shaikh Zayed Medical Complex, Lahore.

\section{Corresponding Author:}

Prof. Ayesha Humayun

Department of Public Health \& Community Medicine, Shaikh Khalifa Bin Zayed Al-Nahyan Medical College, Shaikh Zayed Medical Complex, Lahore.

E-mail: drayeshah@gmail.com 